\title{
Isolation and functional characterization of CE1 binding proteins
}

Sun-ji Lee, Ji Hye Park, Mi Hun Lee, Ji-hyun Yu, Soo Young Kim

\begin{abstract}
Background: Abscisic acid (ABA) is a plant hormone that controls seed germination, protective responses to various abiotic stresses and seed maturation. The ABA-dependent processes entail changes in gene expression. Numerous genes are regulated by ABA, and promoter analyses of the genes revealed that cis-elements sharing the ACGTGGC consensus sequence are ubiquitous among ABA-regulated gene promoters. The importance of the core sequence, which is generally known as ABA response element (ABRE), has been demonstrated by various experiments, and its cognate transcription factors known as ABFs/AREBs have been identified. Although necessary, ABRE alone is not sufficient, and another cis-element known as "coupling element (CE)" is required for full range ABA-regulation of gene expression. Several CEs are known. However, despite their importance, the cognate transcription factors mediating ABA response via CEs have not been reported to date. Here, we report the isolation of transcription factors that bind one of the coupling elements, CE1.

Results: To isolate CE1 binding proteins, we carried out yeast one-hybrid screens. Reporter genes containing a trimer of the CE1 element were prepared and introduced into a yeast strain. The yeast was transformed with library DNA that represents RNA isolated from ABA-treated Arabidopsis seedlings. From the screen of 3.6 million yeast transformants, we isolated 78 positive clones. Analysis of the clones revealed that a group of AP2/ERF domain proteins binds the CE1 element. We investigated their expression patterns and analyzed their overexpression lines to investigate the in vivo functions of the CE element binding factors (CEBFs). Here, we show that one of the CEBFs, AtERF13, confers ABA hypersensitivity in Arabidopsis, whereas two other CEBFs enhance sugar sensitivity.

Conclusions: Our results indicate that a group of AP2/ERF superfamily proteins interacts with CE1. Several CEBFs are known to mediate defense or abiotic stress response, but the physiological functions of other CEBFs remain to be determined. Our in vivo functional analysis of several CEBFs suggests that they are likely to be involved in ABA and/or sugar response. Together with previous results reported by others, our current data raise an interesting possibility that the coupling element CE1 may function not only as an ABRE but also as an element mediating biotic and abiotic stress responses.
\end{abstract}

\section{Background}

Abscisic acid (ABA) is a phytohormone that controls seed germination, seedling growth and seed development [1]. In particular, ABA plays an essential role in the protective responses of plants to adverse environmental conditions, such as drought, high salinity and extreme temperatures [2].

\footnotetext{
* Correspondence: sooykim@chonnam.ac.kr
Department of Molecular Biotechnology and Kumho Life Science Laboratory,

* Correspondence: sooykim@chonnam.ac.kr
Department of Molecular Biotechnology and Kumho Life Science Laboratory, College of Agriculture and Life Sciences, Chonnam National University, Gwangju 500-757, South Korea
}

(c) 2010 Lee et al; licensee BioMed Central Ltd. This is an Open Access article distributed under the terms of the Creative Commons

At the molecular level, ABA-dependent processes entail changes in gene expression patterns. Numerous genes are either up- or down-regulated by ABA in seedlings [3,4]. The ABA regulation of these genes is generally at the transcriptional level, and a number of cis-regulatory elements responsible for the regulation by $\mathrm{ABA}$ have been determined [5]. One of the cis-elements consists of ACGTGGC core sequence. The element, which is similar to the G-box (CACGTG) present in many light-regulated promoters [6], is ubiquitous among ABA-regulated gene promoters and generally known as $A B A$ response element (ABRE). Although necessary, a single copy of the G-box type ABRE 
is not sufficient to mediate $\mathrm{ABA}$ regulation, and multiple copies of ABRE or combinations of ABRE with another cis-element are required for the full ABA-induction of genes. For instance, an element known as CE3 (coupling element 3, ACGCGTGTCCTC) is required for the ABAinduction of barley HVA1 and OsEm genes [7]. Thus, CE3 and $\mathrm{ABRE}$ constitute an ABA response complex. Another coupling element, CE1 (TGCCACCGG), is necessary for the ABA-regulation of HVA22 gene [8]. In RD29A gene, DRE (Dehydration-responsive element, TACCGACAT) functions as a coupling element to ABRE [9].

A subfamily of bZIP proteins has been identified that mediate the ABA response via the G-box type ABRE in Arabidopsis [10,11]. Referred to as ABFs or AREBs, these proteins not only bind the ABRE but also mediate stress-responsive ABA regulation in Arabidopsis seedlings [12]. On the other hand, ABI5, which belongs to the same subfamily of bZIP proteins as ABFs/AREBs $[13,14]$, mediates ABA response in the embryo. ABFs/ AREBs were isolated based on their binding to ABRE. Subsequent study showed that they also bind the coupling element CE3 [10], which is functionally equivalent to ABRE [15].

The transcription factors that bind the CE1 element have not been reported yet. Among the known transcription factors involved in ABA response, ABI4 has been shown to bind the CE1 element [16]. However, the preferred binding site of ABI4 is CACCG, which differs from the CE1 element consensus CCACC. Thus, it has been suggested that AP2 domain proteins other than ABI4 would interact with CE1 [17].

To isolate CE1 element binding factors, we conducted yeast one-hybrid screens. From the screen of 3.6 million yeast transformants, we isolated 78 positive clones. Analysis of the clones revealed that a group of AP2/ERF domain proteins bind the CE1 element in yeast. Most of the CE1 binding factors (CEBFs) belong to the B-3 or the A-6 subfamily of AP2/ERF domain proteins [18,19]. We also found that overexpression of some of the CEBFs alters ABA and/or sugar responses in Arabidopsis.

\section{Results}

\section{Isolation of CE1-binding proteins}

To isolate genes encoding the proteins that bind the CE1 element, we conducted yeast one-hybrid screens [10]. A trimer of the CE1 element was cloned in front of the minimal promoters of the lac Z and the HIS3 reporter genes, respectively. The reporter constructs were then introduced into a yeast strain to create reporter yeast, which was subsequently transformed with cDNA library DNA. The library was prepared from mRNA isolated from ABA-and salt-treated Arabidopsis seedlings. The resulting transformants were screened for reporter activities. From the screen of 3.6 million yeast transformants, we obtained 78 positive clones and analyzed more than 50 clones.

Grouping of the positive clones based on their insert restriction patterns and subsequent DNA sequencing revealed that they encode a group of AP2/ERF superfamily transcription factors (Table 1). Twelve isolates encoded AtERF15 (At2g31230), ten isolates encoded ERF1 (At3g23240) and nine isolates encoded RAP2.4 (At1g78080). Other multiple or single isolate encoded AtERF1 (At4g17500), AtERF5 (At5g47230), AtERF13 (At2g44840) and seven other AP2/ERF family proteins. Among the $13 \mathrm{AP} 2 / \mathrm{ERF}$ proteins isolated, nine belong to the B-3 subfamily, three belong to the A-6 subfamily and one belongs to the B-2 subfamily. Thus, a group of AP2/ERF proteins, especially those belonging to the subgroup B-3, was isolated as CE1-binding factors in our one-hybrid screen. We designated the proteins CEBFs (CE1 binding factors).

\section{DNA-binding and transcriptional activities of CEBFs}

Binding of a number of CEBFs, which were isolated as multiple isolates (Table 1), to the CE1 element was confirmed in yeast. Plasmid DNA was isolated from the positive clones, and their binding to CE1 was determined by investigating their ability to activate the CE1-

Table 1 Results of one-hybrid screen: CE1 element binding factors (CEBFs)

\begin{tabular}{|c|c|c|c|c|}
\hline $\begin{array}{l}\text { No. } \\
\text { isolates }\end{array}$ & Gene ID & $\begin{array}{c}\text { Gene } \\
\text { name }\end{array}$ & $\begin{array}{c}\text { Conserved } \\
\text { domain }\end{array}$ & Group $^{a}$ \\
\hline 12 & At2g31230 & AtERF15 & AP2/ERF & $\begin{array}{c}\text { B-3 } \\
\text { subfamily }\end{array}$ \\
\hline 10 & At3g23240 & ERF1 & $"$ & $\begin{array}{c}\text { B-3 } \\
\text { subfamily }\end{array}$ \\
\hline 4 & At4g17500 & AtERF1 & $"$ & $\begin{array}{c}\text { B-3 } \\
\text { subfamily }\end{array}$ \\
\hline 5 & At5g47230 & AtERF5 & $"$ & $\begin{array}{c}\text { B-3 } \\
\text { subfamily }\end{array}$ \\
\hline 2 & At2g44840 & AtERF13 & $"$ & $\begin{array}{c}\text { B-3 } \\
\text { subfamily }\end{array}$ \\
\hline 1 & At5g47220 & AtERF2 & $"$ & $\begin{array}{c}\text { B-3 } \\
\text { subfamily }\end{array}$ \\
\hline 1 & At5g07580 & - & $"$ & $\begin{array}{c}\text { B-3 } \\
\text { subfamily }\end{array}$ \\
\hline 1 & At1g06160 & ORA59 & $"$ & $\begin{array}{c}\text { B-3 } \\
\text { subfamily }\end{array}$ \\
\hline 1 & At5g61590 & - & $"$ & $\begin{array}{c}\text { B-3 } \\
\text { subfamily }\end{array}$ \\
\hline 2 & At1g53910 & RAP2.12 & $"$ & $\begin{array}{c}\text { B-2 } \\
\text { subfamily }\end{array}$ \\
\hline 9 & At1g78080 & RAP2.4 & $"$ & $\begin{array}{c}\text { A-6 } \\
\text { subfamily }\end{array}$ \\
\hline 2 & At1g22190 & RAP2.4L & $"$ & $\begin{array}{c}\text { A-6 } \\
\text { subfamily }\end{array}$ \\
\hline 2 & At4g13620 & - & $"$ & $\begin{array}{c}\text { A-6 } \\
\text { subfamily }\end{array}$ \\
\hline
\end{tabular}

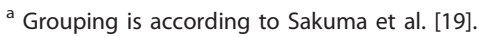


containing lac $\mathrm{Z}$ reporter gene. Figure $1 \mathrm{~A}$ shows the results obtained with six different positive clones: AtERF15, AtERF5, AtERF1, AtERF13, RAP2.4 and RAP2.12. The four AtERFs, which belong to the B-3 subfamily, could activate the reporter gene containing the CE1 element but not the reporter gene lacking the CE1 element. The CE1-dependent reporter activation was observed with medium containing galactose but not with the medium containing glucose. Thus, reporter activation was also dependent on the presence of galactose, which is an inducer of the GAL1 promoter that drives the expression of the cDNA clones. Similarly, RAP2.12 and RAP2.4, which belong to the B-2 and the A-6 subfamily, respectively, could also activate the reporter gene, and the activation was CE1- and galactose-dependent.

CEBFs are putative transcription factors; accordingly, we wanted to determine if they possess transcriptional activity. To accomplish this, the transcriptional activity of CEBFs was examined employing a yeast assay system.

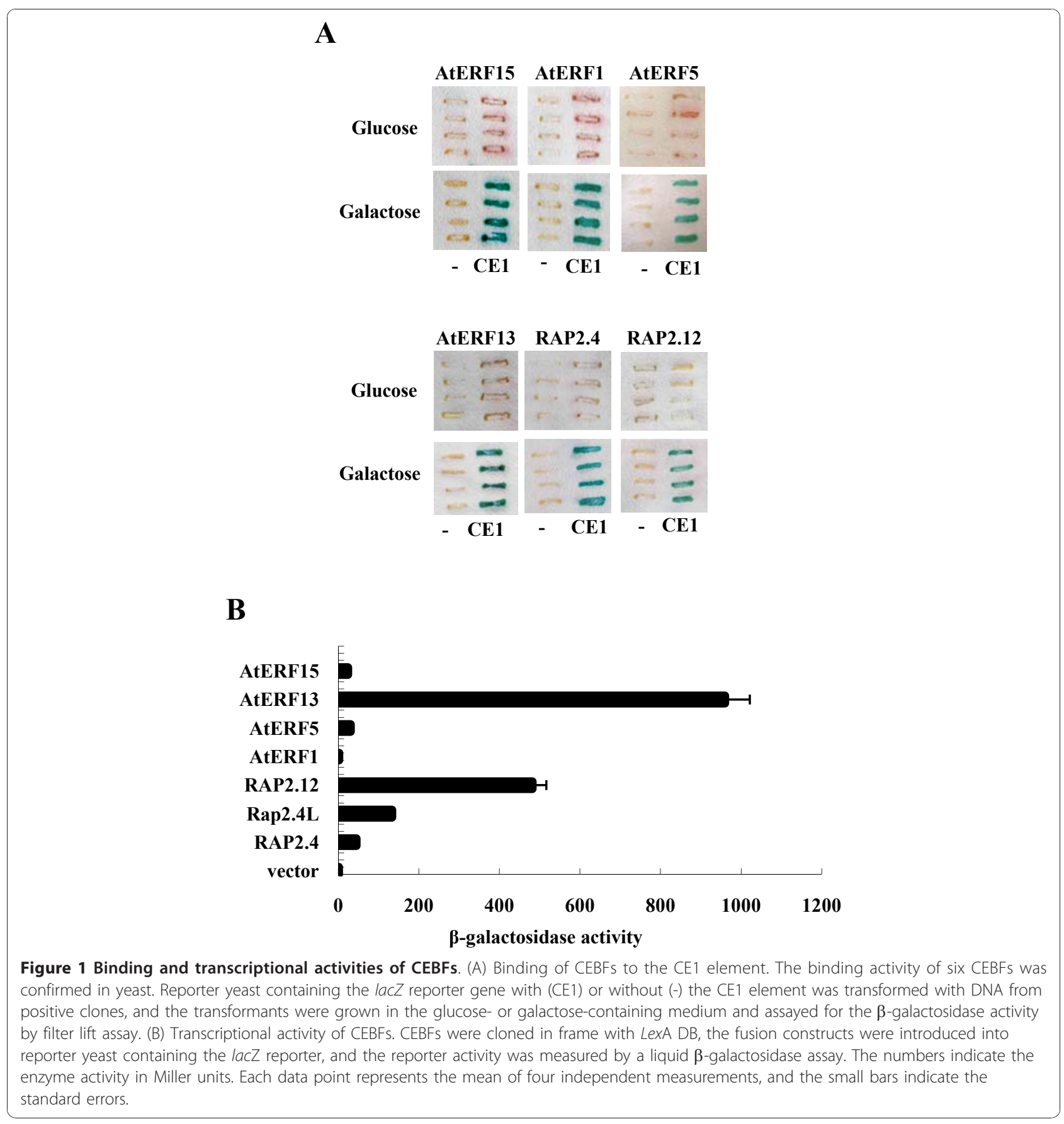


The coding regions of seven CEBFs were individually cloned in frame with the $\operatorname{Lex} \mathrm{A} D B$ in the vector pPC62LexA [20]. The hybrid constructs were then introduced into the yeast strain L40, which carries a lac $\mathrm{Z}$ reporter gene with an upstream LexA operator in its promoter. Figure 1B shows that AtERF13 has the highest transcriptional activity among the seven CEBFs. RAP2.12 also possesses high transcriptional activity, while RAP2.4, RAP2.4L (At1g22190), AtERF5 and AtERF15 displayed relatively lower transcriptional activity. AtERF1 was found to have very low transcriptional activity.

\section{Expression patterns of CEBFs}

The expression patterns of nine CEBFs in seedlings were examined by coupled reverse transcription and polymerase chain reaction (RT-PCR). Because the tissue-specific expression patterns of many AP2/ERF domain proteins have been reported [21], we focused on the ABA and stress induction patterns of CEBFs. Figure 2A shows that the expression of AtERF1, AtERF2, AtERF13 and AtERF15 was induced by high salt. In the case of AtERF13, its expression was also induced by high osmolarity (i.e., mannitol). The expression of other CEBFs was largely constitutive or their induction levels were very low.

For AtERF13, RAP2.4 and At1g22190, which was designated RAP2.4L (RAP2.4-like) because of its high similarity to RAP2.4, we examined their tissue-specific expression patterns in detail by investigating their promoter activity. Transgenic plants harboring the promoter-GUS reporter constructs were prepared, and histochemical GUS staining was carried out to determine their temporal and spatial expression patterns.

With ATERF13, GUS activity was observed only in the shoot meristemic region and the emerging young leaves in seedlings (Figure 2B). Thus, AtERF13 expression in seedlings was specific to the shoot meristem region. During the reproductive stage, GUS activity was observed in the carpels. On the other hand, GUS activity was observed in most of the tissues with the RAP2.4L promoter (Figure 2C). GUS activity was not observed in the immature embryo, but it is detected in the mature embryo and most of the seedling tissues. The GUS activity was strong in most of the tissues, although relatively weaker activity was observed in young leaves and the lateral root tips including the meristem and the elongation zone. Strong GUS activity was also observed in reproductive organs such as sepals, filaments, style and abscission zone. The GUS staining pattern of the transgenic plants harboring the RAP2.4 promoter construct was similar to that of the plants harboring the RAP2.4L promoter construct (Figure 2D). In general, stronger GUS activity was observed with the RAP2.4 promoter, and, unlike the RAP2.4L promoter, its activity was detected in the emerging young leaves.

To obtain further clues to the function of AtERF13, RAP2.4L and RAP2.4, we determined their subcellular localization. The coding regions of the CEBFs were individually fused to EYFP under the control of the $35 \mathrm{~S}$ promoter, and the localization of the fusion proteins was examined after Agroinfiltration of tobacco leaves. Figure 2E shows that YFP signal is detected in the nucleus with the AtERF13 construct. Similarly, the YFP signal was also observed in the nucleus with RAP2.4L and RAP2.4. Thus, our results indicate that AtERF13, RAP2.4L and RAP2.4 are localized in the nucleus.

\section{In vivo functions of CEBFs}

Our transcriptional assay (Figure 1B) showed that AtERF13 has the highest transcriptional activity among CEBFS, and its expression was highly inducible by high salt (Figure 2A). Hence, we chose AtERF13 for functional analysis. To determine the in vivo function of AtERF13, we generated its overexpression (OX) lines. The coding region of AtERF13 was fused to the CaMV $35 \mathrm{~S}$ promoter employing the pBI121 vector [22], and after transformation of Arabidopsis, T3 or T4 generation transgenic plants were recovered and used for phenotype analysis.

AtERF13 OX lines exhibited minor growth retardation (Figure 3A), and mature plants were slightly smaller than the wild type plants (not shown). However, other than minor dwarfism, the overall growth pattern was normal. Because the CE1 element is an ABA response element, we determined the ABA-associated phenotypes to address whether AtERF13 overexpression affected ABA response. The germination rates of the transgenic plants were slightly slower $(\sim 2 \mathrm{hr})$ in ABA-free medium (not shown), but the ABA sensitivity of the transgenic seed germination was similar to that of the wild type plants (not shown).

Unlike the seed germination, postgermination growth of the AtERF13 OX lines exhibited altered ABA response. Figure $3 \mathrm{~B}$ and Figure $3 \mathrm{C}$ show that shoot development of the transgenic plants was inhibited severely at low concentrations of ABA. For instance, cotyledons of less than $50 \%$ of the transgenic plants turned green at $0.5 \mu \mathrm{M} \mathrm{ABA}$, and true leaf development was not observed with any of the transgenic plants. By contrast, shoot development of wild type seedlings was not affected significantly by the same concentration of ABA. Similarly, root growth of the AtERF13 OX lines was also severely inhibited at $0.5 \mu \mathrm{M} A B A$, whereas root growth of the wild type plants was affected much less (Figure 3D). Thus, postgermination growth of the AtERF13 OX lines was hypersensitive to ABA. 
B

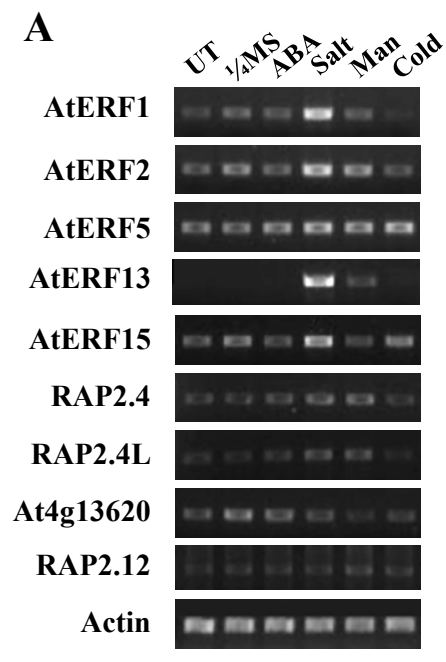

c

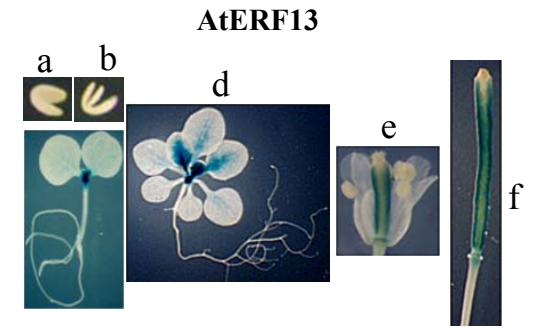

C

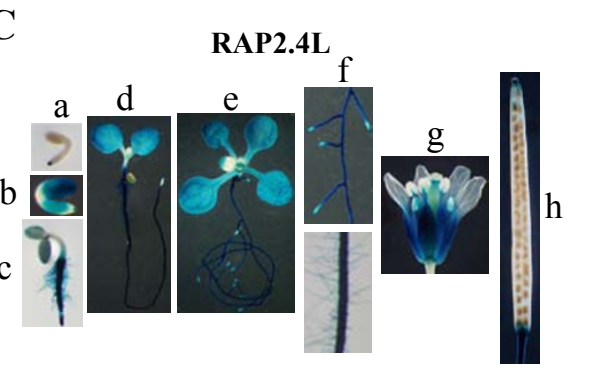

D

RAP2.4

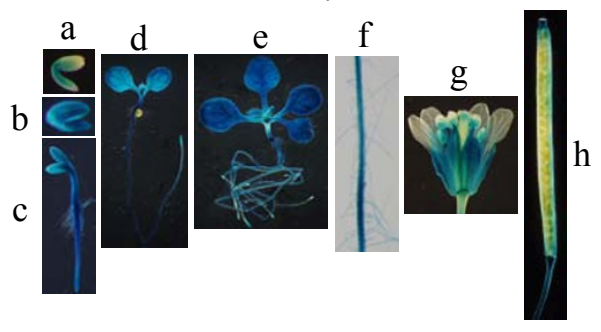

$\mathbf{E}$

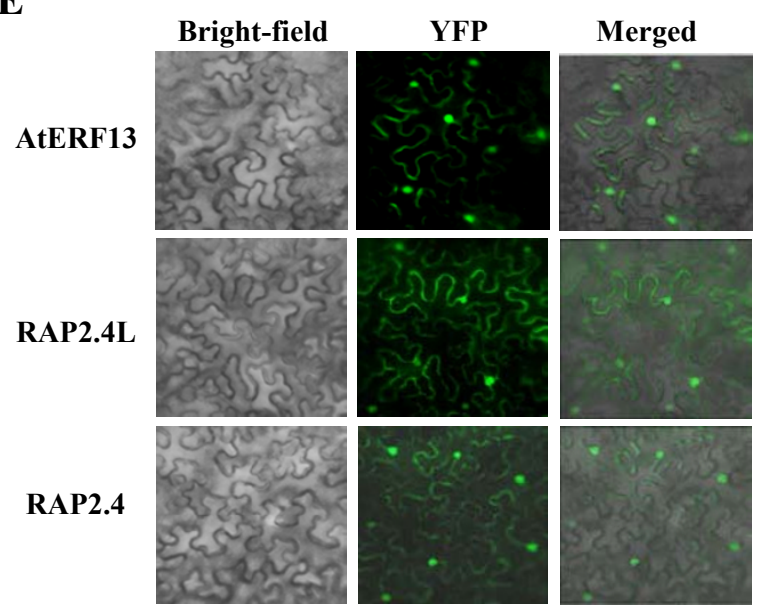

Figure 2 Expression patterns of CEBFs. (A) Induction patterns of CEBFs were determined by RT-PCR. UT, untreated plants. Plants were treated with 1/4MS, ABA, NaCl (Salt), $600 \mathrm{mM}$ mannitol (Man) for 4 hrs, or placed at $4 \mathrm{C}$ for $24 \mathrm{hr}$ (Cold) before RNA was isolated. (B) Histochemical GUS staining of transgenic plants harboring AtERF13 promoter-GUS reporter gene construct. a. immature embryo. b. mature embryo. c, 5-day-old seedling. $d$, 15-day-old seedling. e, flower. f, immature silique. (C) Histochemical GUS staining of transgenic plants harboring the RAP2.4L promoter-GUS reporter gene construct. a. mature embryo. b. mature embryo. c, 2-day-old seedling. d, 5-day-old-seedling. e, 14-day-old seedling. f, roots of 14-day-old seedling. g, flower. h, mature silique. (D) Histochemical GUS staining of transgenic plants harboring RAP2.4 promoter-GUS reporter gene construct. a. mature embryo. b. mature embryo. c, 2-day-old seedling. d, 5-day-old-seedling. e, 14-day-old seedling. f, roots of 14day-old seedling. g, flower. h, mature silique. In B-D, GUS staining was conducted for 20 hrs. (E) Subcellular localization of AtERF13, RAP2.4L and RAP2.4. Tobacco leaves were infiltrated with Agrobacterium as described in the Methods and observed with fluorescence microscope 40 hrs after infiltration. Bright field, fluorescence (YFP) and merged images of the tobacco leaves are shown. 


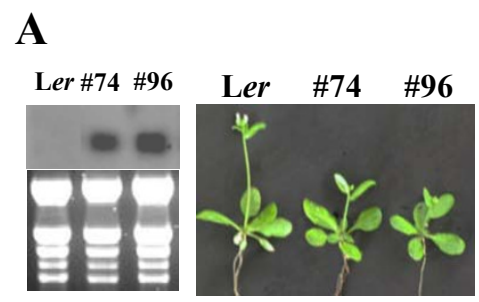

C

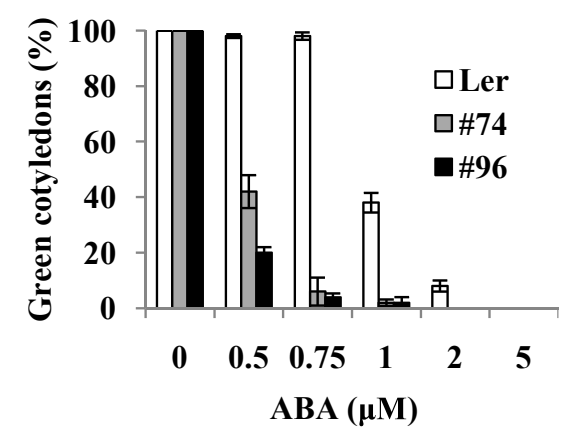

$\mathbf{E}$

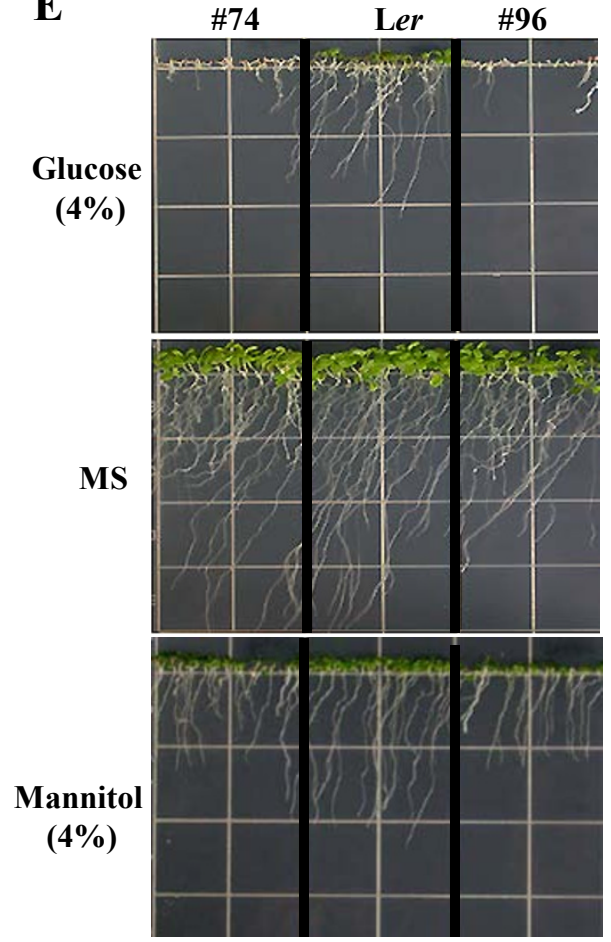

B

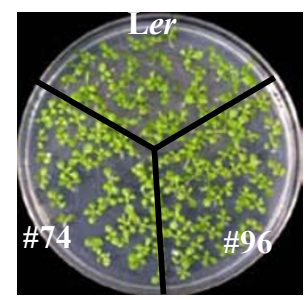

MS

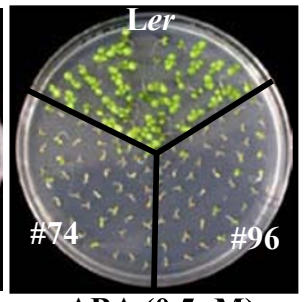

ABA (0.5 $\mu M)$

D

ABA

$(0.5 \mu \mathrm{M})$

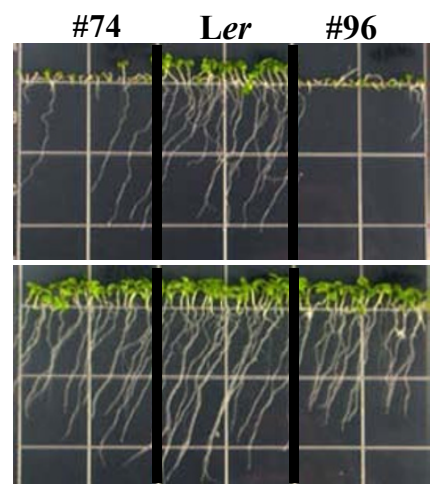

F

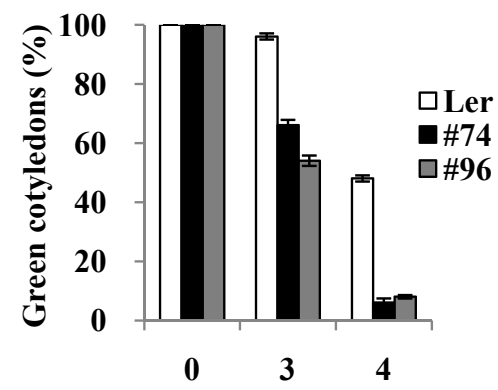

Glucose (\%)

Figure $3 \mathrm{ABA}$ and glucose sensitivity of AtERF13 overexpression lines. (A) Growth of AtERF13 OX lines. Three-week-old plants grown in soil. The numbers indicate line no. and the left panel shows the AtERF13 expression levels determined by Northern analysis. (B) Growth of the OX lines in the presence of $0.5 \mu \mathrm{M}$ ABA. Seeds were germinated and grown for 10 days. (C) ABA dose response of shoot development measured by cotyledon greening efficiency. Seeds were germinated and grown for 11 days on MS medium containing various concentrations of ABA, and seedlings with green cotyledons were counted. Experiments were done in triplicates ( $n=50$ each), and the small bars indicate standard errors. (D) Root growth of the OX lines in the presence of $0.5 \mu \mathrm{M}$ ABA. (E) Growth of the OX lines in the presence of $4 \%$ glucose. (F) Glucose dose response determined by cotyledon greening. Plants were grown on MS medium containing 3 or $4 \%$ glucose for 11 days before counting seedlings with green cotyledons. Experiments were conducted in triplicates $(n=50$ each). The small bars represent standard errors. 
We next examined the glucose sensitivity of the AtERF13 OX lines. Glucose inhibits shoot development, i.e., cotyledong greening and true leaf development, and the inhibition process is mediated by ABA [23]. Figure $3 \mathrm{E}$ and Figure 3F show that glucose-dependent arrest of shoot development was much more severe in the AtERF13 OX lines. At 3\% glucose, cotyledon greening of the wild type plants was not affected noticeably. By contrast, cotyledon greening efficiency of the transgenic plants was reduced to approximately $50 \%$. At $4 \%$ glucose, shoot development was observed with approximately $50 \%$ of the wild type plants, whereas less than $10 \%$ of the OX lines develop green cotyledons. Thus, our results indicated that AtERF13 OX lines are hypersensitive to glucose. We did not observe changes in mannitol (Figure 3E) or salt (Additional file 1) response in parallel experiments, suggesting that the effect is glucose-specific.

We conducted similar experiments to investigate the in vivo function of RAP2.4L, which belongs to the A-6 subfamily and whose function has not been reported yet. RAP2.4L OX lines were constructed, and their phenotypes were scored to address its involvement in ABA response. The transgenic plants displayed minor growth retardation (Figure 4A), but no distinct changes in ABA sensitivity were observed. On the other hand, the RAP2.4L OX lines displayed altered response to glucose. Figure 4B and Figure 4C show that shoot development of the RAP2.4L OX lines was more severely inhibited by $3 \%$ and $4 \%$ glucose than the wild type plants. As mentioned above, RAP2.4 is highly homologous to RAP2.4L. Therefore, we prepared RAP2.4 OX lines and analyzed their phenotypes as well (see Discussion). We did not observe changes in ABA sensitivity; however, similar to RAP2.4L OX lines, the RAP2.4 OX lines were hypersensitive to glucose (Figure 4B and Figure 4D). We also examined the salt tolerance of RAP2.4L and RAP2.4 OX lines. The results showed that postgermination growth, i.e., cotyledon greening and true leaf development of both transgenic lines was more severely inhibited at 125 and $150 \mathrm{mM} \mathrm{NaCl}$ than wild type plants. The salt sensitivity of RAP2.4 OX lines was more pronounced than that of RAP2.4L. We did not observe changes in mannitol sensitivity in either the RAP2.4 or the RAP2.4L OX lines (Additional file 2).

To further confirm their involvements in ABA response, we analyzed knockout lines of RAP2.4L and RAP2.4 and RNAi lines of AtERF13. We did not observe distinct phenotypes with the transgenic plants, presumably because of the functional redundancy among CEBFs.

To investigate the target genes of AtERF13, we determined the changes in the expression levels of a number of ABA-responsive genes by Real-Time RT-PCR.
Among the genes we investigated, the expression level of COR15A increased significantly in the AtERF13 OX lines (Figure 5). Slight increases in $A D H 1$ expression were also observed. By contrast, RAB18 expression decreased or increased slightly in the transgenic lines. Similar analysis showed that COR15A and ADH1 expression levels were enhanced in the RAP2.4L and the RAP2.4 OX lines. Increase in the RAB18 expression level was also observed in the RAP2.4 OX line (\#3). The three genes whose expression levels were altered in the transgenic lines have the G-box type ABREs in their promoter regions and are inducible by both $A B A$ and various abiotic stresses. Additionally, COR15A and $R A B 18$ genes have a sequence element (i.e., CCGAC) that can function as another coupling element, DRE, although the CE1 core sequence, CCACC, was not found.

\section{Discussion}

We isolated genes encoding CE1 element binding factors (CEBFs) employing a yeast one-hybrid system. CEBFs belong to the AP2/ERF superfamily of transcription factors $[18,19]$. The AP2/ERF proteins are classified into three families: AP2, ERF and RAV. Whereas AP2 and RAV family members possess an additional AP2 or B3 DNA-binding domain, ERF family members possess a single AP2/ERF domain. The ERF family is further divided into two subgroups, the DREB/CBF subfamily (group A) and the ERF subfamily (group B) [19]. Among the 52 positive clones we analyzed, 39 encoded B group proteins (i.e., B-3 subfamily members), whereas 13 encoded A group proteins (i.e., A-6 subfamily members) (Table 1).

The in vitro binding sites of many AP2/ERF superfamily proteins have been studied in detail. The DRE core sequence, i.e., the binding site for DREB1A and DREB2A, which are representative members of the DREB/CBF subfamily, is A/GCCGAC [19]. The GCC box core sequence, which is the consensus binding site for ERF family members, is AGCCGCC [24]. Thus, the two sequences share the CCGNC consensus sequence, the central G being essential for high affinity binding. On the other hand, the core sequence of the CE1 element is CCACC, which differs from the DRE and the GCC box core sequences. The results of our one-hybrid screen indicate that a subset of AP2/ERF family members (i.e., at least ten B-3/B-2 subgroup members and three A- 6 subgroup proteins) bind the CE1 element in yeast.

Several of the CEBFs have been reported as GCC box binding proteins. For example, the preferred in vitro binding site of AtERF1, AtERF2 and AtERF5 is the wild type GCC box, AGCCGCC [25]. Mutations of the Gs at the second and fifth positions reduced their binding activity to less than $10 \%$ of that obtained with the wild 


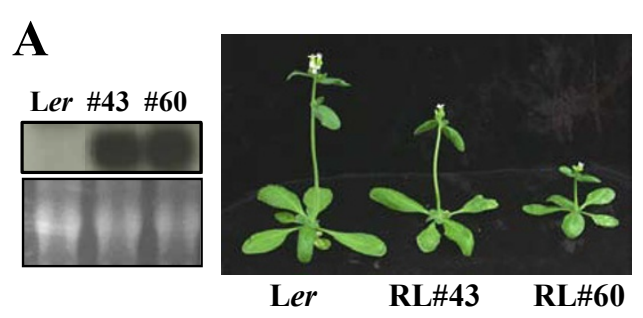

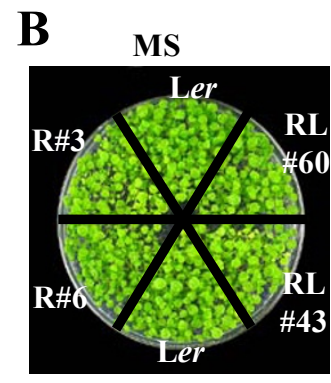

C

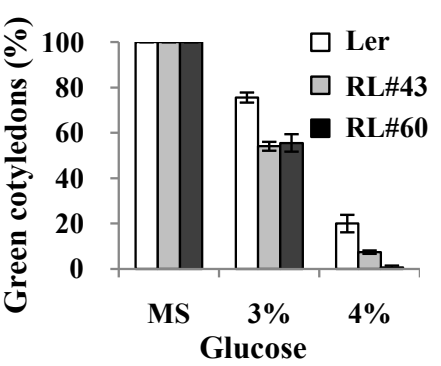

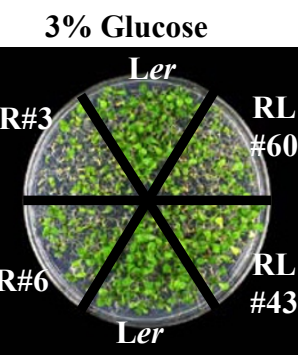

D
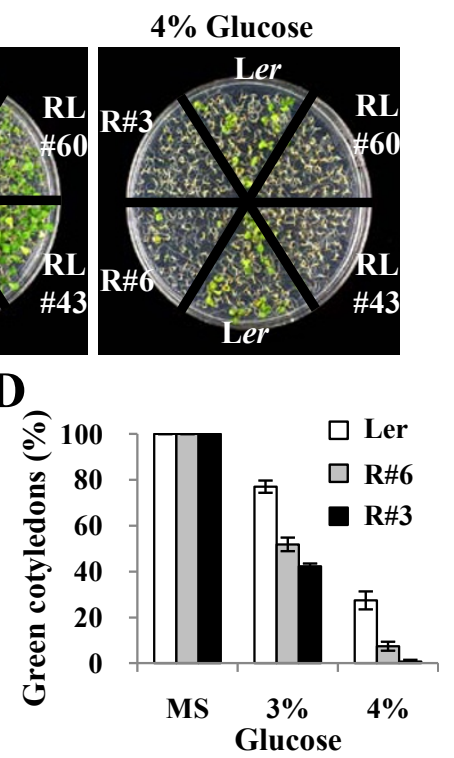

$\mathbf{E}$
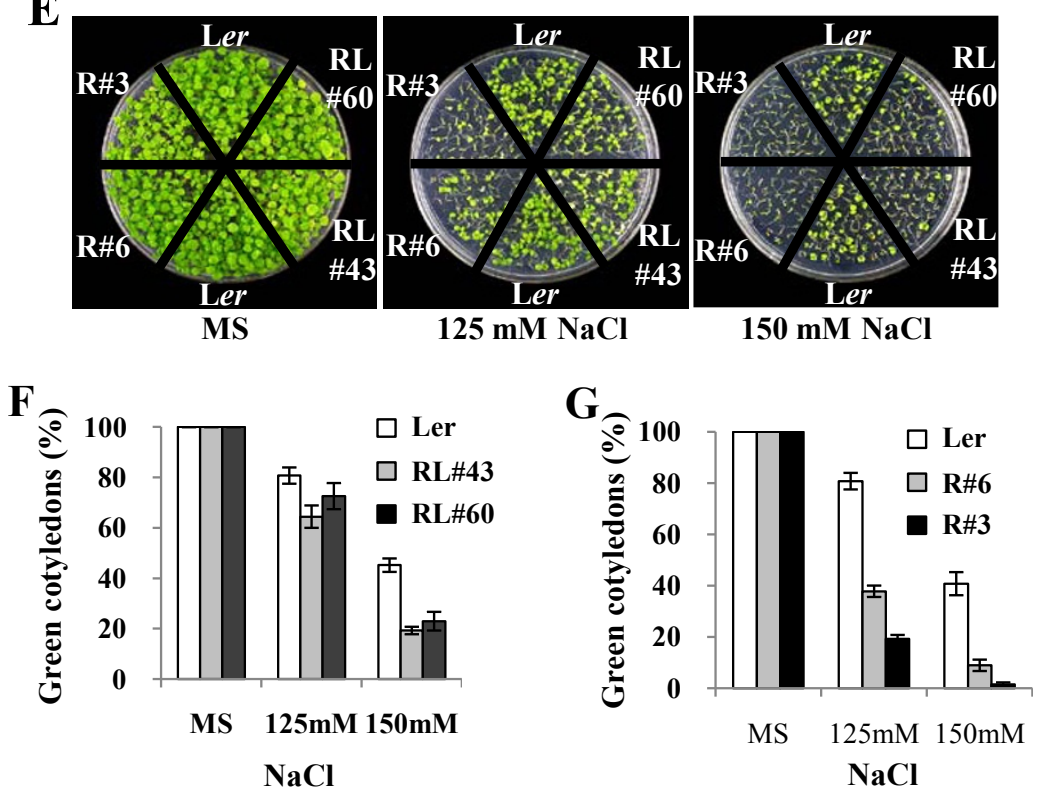

Figure 4 Glucose and salt sensitivity of RAP2.4L overexpression lines. (A) Growth of RAP2.4L OX lines. Plants were grown in soil for five weeks. The left panel shows the RAP2.4L expression levels in the transgenic lines (\#43 and \#60) determined by Northern analysis. (B) Plants grown in the presence of $3 \%$ or $4 \%$ glucose for 13 days. R, RAP2.4. RL, RAP2.4L. The numbers indicate line numbers. (C) Glucose dose response of RAP2.4L OX lines. (D) Glucose dose response of RAP2.4 OX lines. (E) Plants grown in the presence of salt for 13 days. (F) Salt dose response of RAP2.4L OX lines. (G) Salt dose response of RAP2.4 OX lines. In (C), (D), (F) and (G), experiments were conducted in triplicates $(n=45$ each), and the small bars indicate the standard errors. 


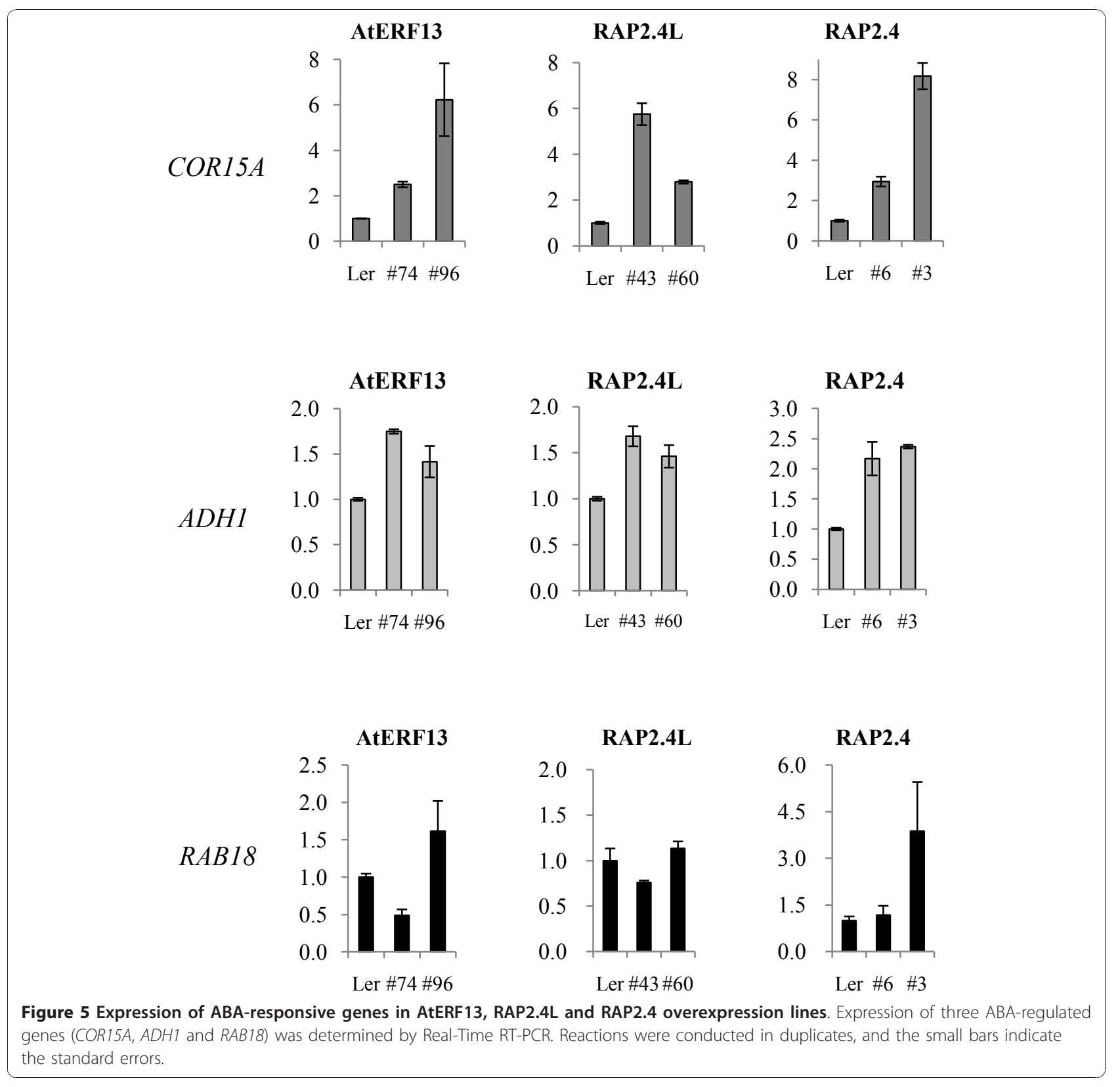

type sequence. Similarly, the mutation of the second G of the core sequence greatly reduced the in vitro binding of RAP2.4 [26]. However, in our one-hybrid screen, AtERF1, AtERF5 and RAP2.4 were isolated as multiple isolates (i.e., 4, 5 and 9 isolates, respectively). The result suggests that these proteins can interact with the nonGCC box sequence, CCACC, under physiological conditions (i.e. in yeast).

$\mathrm{AP} 2 / \mathrm{ERF}$ proteins are involved in various cellular processes, including biotic and abiotic stress responses $[18,19]$. Many DREB/CBF family proteins (e.g., DREB1A, DREB1B, DREB1C, DREB2A, RAP2.1 and RAP2.4) are involved in ABA-independent abiotic stress responses
[19,26,27], whereas ERF family members (e.g., ERF1, ORA59, AtERF2, AtERF4, AtERF14, and RAP2.3) are generally involved in ethylene and pathogen defense responses [18,28-34]. In particular, several of the AP2/ ERF proteins are involved in ABA response. ABI4, which belongs to the DREB/CBF subfamily, is a positive regulator of $\mathrm{ABA}$ and sugar responses [35]. DREB2C and maize DBF1 are also positive regulators of $A B A$ response [36,37]. On the other hand, AtERF7 [38], ABR1 [39] and AtERF4 [34] are ERF subfamily proteins that are negative regulators of $\mathrm{ABA}$ response.

To determine the in vivo functions of CEBFs in ABA response, we generated their $\mathrm{OX}$ lines and acquired 
knockout lines for phenotype analysis when available. As mentioned above, several CEBFs (i.e., ERF1, AtERF2 and ORA59) are known to regulate defense responses. However, their involvement in ABA response and the functions of other CEBFs have not been reported yet. Here, we present our results obtained with CEBFs, AtERF13 and RAP2.4L. AtERF13 was found to possess very high transcriptional activity in yeast (Figure 1B) and localized in the nucleus. Its expression was limited to the shoot meristem region and young emerging leaves (Figure 2B), implying that it may play a role in shoot growth or development. Consistent with this notion, AtERF13 OX lines exhibited minor dwarfism (Figure 3A). The growth retardation observed in the OX lines may reflect the normal inhibitory role of AtERF13 or be the result of its ectopic overexpression. However, we think that AtERF13 probably play a role in growth regulation. Because we could not obtain its knockout lines, we prepared and analyzed its RNAi lines. Our results showed that the RNAi lines grew faster than wild type plants (Additional file 3), suggesting that AtERF13 may inhibit seedling growth.

Overexpression of AtERF13 conferred ABA hypersensitivity during postgermination growth. As shown in Figure 3 both shoot and root growth was severely inhibited by the low concentration of ABA, which had little effect on wild type seedling growth. Additionally, the AtERF13 OX lines were hypersensitive to glucose, whose effect is mediated by ABA. We did not carry out extensive expression analysis of ABA-responsive genes in AtERF13 OX lines. However, our limited target gene analysis showed that expression of several ABA-responsive genes was affected by AtERF13 (Figure 5). Thus, our results strongly suggest that AtERF13 may be involved in ABA response. As mentioned in the Results, we did not observe distinct phenotypes with AtERF13 RNAi lines except faster seedling growth, presumably because of the functional redundancy among CEBFs.

In the case of RAP2.4L, we did not observe changes in ABA sensitivity in its OX lines, although we observed up-regulation of several ABA-responsive genes (Figure 5). However, the transgenic lines were glucose-hypersensitive, suggesting that it may be involved in sugar response (Figure 4B). We also analyzed its knockout lines, but did not observe distinct phenotypes (not shown). RAP2.4 is the closest homologue of RAP2.4L; therefore, we also analyzed its OX and knockout phenotypes. We did not observe alterations in ABA response in either the OX or the knockout lines of RAP2.4 (not shown). The results are consistent with those observed by Lin et al. [26], who reported that RAP2.4 is involved in light, ethylene and ABA-independent drought tolerance but not in ABA response. However, similar to RAP2.4L OX lines, RAP2.4 OX lines were glucose- sensitive and both RAP2.4 and RAP2.4L OX lines were salt-sensitive (Figure 4E-4G). Additionally, single or double knockout lines of RAP2.4 and RAP2.4L grew faster than wild type plants (Additional file 3), suggesting their role in seedling growth control.

It is not known whether other CEBFs are involved in ABA response. Another important question to be addressed is the mechanism of their function, if they are involved in $\mathrm{ABA}$ response. $\mathrm{CE} 1$ constitutes an $\mathrm{ABA}$ response complex with the G-box type $A B R E$ and functions in combination with ABRE. Therefore, CEBFs are expected to interact with the transcription factors ABFs/ AREBs, which mediate ABA response in seedlings via the G-box type ABRE. In the case of DREB2C, which binds another coupling element DRE, its physical interaction with ABFs/AREBs has been demonstrated [37]. It would be worthwhile to determine whether CEBFs can physically interact with ABFs/AREBs. As described before, several CEBFs mediate plant defense response. Thus, our results raise an interesting possibility that $\mathrm{CE} 1$ may be a converging point of $\mathrm{ABA}$ and defense responses.

\section{Conclusions}

We conducted one-hybrid screen to isolate proteins that interact with the coupling element CE1 and isolated a group of AP2/ERF superfamily proteins designated as CEBFs. To determine the function of CEBFs, we examined their expression patterns and prepared OX lines for phenotype analysis. Our results showed that the AtERF13 OX lines are ABA-and glucose-hypersensitive. The OX lines of two other CEBFs, RPA2.4 and RAP2.4L, were glucose-hypersensitive. Thus, overexpression of the three CEBFs resulted in alterations in ABA and/or sugar response. In addition, several ABA-regulated genes were up-regulated in the transgenic lines. Taken together, our data strongly suggest that the three CEBFs evaluated in this study are involved in ABA or stress response. The functions of other CEBFs remain to be determined.

\section{Methods}

\section{One-hybrid screen}

One-hybrid screen was conducted as described before [10]. To prepare reporter gene constructs, a trimer of the oligonucleotides, 5'-CATTGCCACCGGCCC-3', and its complementary oligonucleotides were annealed and cloned into the Zero Blunt TOPO (Invitrogen) vector. The insert was then excised out by Spe I-Eco RV or Kpn I-Xho I digestion. The fragments were then cloned into pSK1, which was prepared by Bam HI digestion followed by Klenow treatment and Spe I digestion, and Kpn I-Xho I digested pYC7-I, respectively. The reporter constructs were sequentially introduced into YPH500 to prepare reporter yeast harboring HIS3/lacZ double 
reporters. Yeast transformation was carried out as described before [10], using the cDNA library DNA representing mRNA isolated from Arabidopsis seedlings treated with ABA and salt.

Approximately 3.6 million yeast transformants were screened, and 78 positive clones were isolated. The positive clones were grouped according to the restriction patterns after EcoR1 and/or Hae III digestion of the insert DNA, which was prepared by PCR. Plasmid DNA was rescued from the representative clones of each group and other non-grouped clones and sequenced. Fifty two positive clones were analyzed and sequenced. For the confirmation test shown in Figure 1A, plasmid DNA from the positive clones was re-introduced into the yeast reporter strain and activation of the lac Z reporter was examined by a filter lift assay.

\section{Transcriptional assay}

Transcriptional activity was determined employing a yeast system, as described previously [20]. The coding regions of CEBFs were prepared by PCR and individually cloned into the Sma I-Not I sites of pPC62/LexA bait vector containing LexA DB. Primer sequences are available upon request. The bait constructs were subsequently introduced into L40 (MAT $\alpha$, his $3 \Delta 200$, trp1901, leu2-3112, ade2, LYS2::[LexAop(x4)-HIS3], URA3:: LexAop(x8)-LacZ, GAL4) (Invitrogen, USA), which carries a lacZ reporter gene with LexA binding sites in its promoter. $\beta$-galactosidase activity was measured by liquid assay using ONPG (O-nitrophenyl- $\beta$-D-galactopyranoside) as a substrate and expressed in Miller units.

\section{RNA isolation and expression analysis}

RNA was isolated employing the RNeasy plant mini kit (Qiagen, USA). Northern blot analysis was carried out, as described previously [20]. For RT-PCR analysis, RNA was treated with DNase I to remove possible contaminating DNA before cDNA synthesis, and the first strand cDNA was synthesized using Superscript III (Invitrogen) according to the manufacturer's instructions. cDNA amplification was carried out within a linear range using genespecific primers. For quantitative RT-PCR, the cDNA was diluted 10-fold, and PCR was performed with SsoFast EvaGreen supermix in a Bio-Rad CFX96 Real-Time PCR Systems (Bio-Rad) according to the supplier's instructions. Quantitation was carried out using the CFX96 Real-Time PCR Systems software, employing actin-1 as a reference gene. Primer sequences are available upon request.

\section{Determination of promoter activity and subcellular localization}

To prepare promoter-GUS constructs, approximately 2.5 kb 5' flanking sequences of AtERF13, RAP2.4 and RAP2.4L were prepared and cloned into pBI101.2 [22].
For AtERF13, the promoter fragment was amplified from genomic DNA using the primer set 5'-AAG CTT GGT ACT AGT ACT GCT AGG TTT CTC-3' and 5'AAT GGA TTC TTG AAT GCT TCT GAA-3'. The resulting fragment was digested with Hind III and then ligated with PBI101.2, which was predigested with Hand III and Sma I. For RAP2.4 and RAP2.4L, the primer sets, 5'-acg cgtc gac CAT CCC TGT ACC ACT CAC TAT CTT ATT C -3' and 5'-GAA TCC GAA AAA ATT GAA CCT GAG AC-3', and 5'-acg cgt cga cTA ACA CAC AAA ATG TAC CGA AAG AAG-3' and 5'CTG TGT AGA TTT CTG AGA GGA GGG A-3' were employed to amplify the promoter fragments. The PCR products were then digested with Sal I and ligated with pBI101.2 cut with Sal I-Sma I. Transformation of Arabidopsis plants (Ecotype, Landsberg erecta, Ler) were according to Bechtold and Pelletier [40]. Histochemical GUS staining was conducted as describe before [41], using T2 or T3 generation plants.

To investigate the subcellular localization, the coding regions of AtERF13, RAP2.4 and RAP2.4L were fused with the EYFP coding region of $\mathrm{p} 35 \mathrm{~S}-\mathrm{FAST} / \mathrm{EYFP}$ in frame. The coding region of AtERF13 was prepared by PCR using the primers 5'-aag ccc ggg ATG AGC TCA TCT GAT TCC GTT AAT-3' and 5'- aag cCc ggg TAT CCG ATT ATC AGA ATA AGA ACA TT-3', and the amplified fragment was digested with Xma I prior to ligation with Xma I-cut p35S-FAST/EYFP. The coding region of RAP2.4 was amplified using the primer set 5'aag gag ctc ATG GCA GCT GCT ATG AAT TTG TAC-3' and 5'- aag ccc ggg AGC TAG AAT CGA ATC CCA ATC GAT-3', whereas the coding region of RAP2.4L was amplified using the primers 5'- aag gag ctc ATG ACA ACT TCT ATG GAT TTT TAC AG-3' and 5'-aag ccc ggg ATT TAC AAG ACT CGA ACA CTG AAG-3'. The amplified fragments were treated with Sac I and Sma I and subsequently ligated with p35S-FAST/ EYFP digested with the same enzymes.

Agrobacterium infiltration was according to Witt et al. and Voinnet et al. [42,43]. Tobacco (Nicotiana benthamiana) leaves were co-infiltrated with the Agrobacterium strains (C58C1) containing the above constructs and p19, respectively. The images of the tobacco epidermal cells were taken with the Olympus BX51 microscope with a YFP filter $40 \mathrm{hr}$ after infiltration.

\section{Generation of transgenic plants and phenotype analysis}

To prepare OX vector constructs, the coding regions of AtERF13, RAP2.4 and RAP2.4L were amplified from a cDNA library and cloned into pBI121. The RAP2.4 coding region was amplified using the primers 5'- TAG GAT CCA TGG CAG CTG CTA TGA ATT TGT ACA CTT G-3' and 5'- TTG CCC CTA AGC TAG AAT CGA ATC CCA ATC-3'. The RAP2.4L coding region was amplified 
using the primers 5'- CCG GAT CCA TGA CAA CTT CTA TGG ATT TTT ACA GT-3' and 5'- CAA CAT CTA ATT TAC AAG ACT CGA ACA CT-3'. The amplified fragments were digested with Bam $\mathrm{HI}$ and cloned into pBI121, which was prepared by the removal of the GUS coding region after Bam HI-Eco ICRI digestion. The coding region of AtERF13 was prepared by PCR using the primer set 5'- CGT CTA GAA TGA GCT CAT CTG ATT CCG TTA ATA ACG G-3' and 5' - AAC TAA TTA TAT CCG ATT ATC AGA ATA AG-3'. The fragment was treated with Xba I and ligated with GUSless pBI121, which was prepared by removal of the GUS coding region after Xba I-Eco ICRI digestion.

For the AtERF13 RNAi construct, the primers 5'-GGG GCG CGC CGC ATT TGA TTG GTT CTT GTA AGT ATG AG-3' and 5'- CGT AAA TTT ATA CTA TGG AAC CGA ATT TAG AAG-3' were used to amplify the $387 \mathrm{bp}$ sense orientation fragment. The fragments were cloned into pFGC5941 after Asc I-Swa I digestion. Primers 5'- GGT CTA GAG CAT TTG ATT GGT TCT TGT AAG TAT GAG-3' and 5'- CGG GAT CCT ACT ATG GAA CCG AAT TTA GAA G-3' were employed to amplify the antisense fragment, which was cloned into pFGC5941 containing the sense fragment after Bam HI-Xba I digestion. The intactness of the cloned sequences of all of the constructs used in this study was confirmed by DNA sequencing.

Arabidopsis transformation was carried out as described above. More than ten transgenic lines were recovered for each CEBF, and $\mathrm{T} 3$ or $\mathrm{T} 4$ generation homozygous lines were employed for phenotype analysis, which was carried out as described before [41].

Seeds of knockout lines, SALK_093377 and SALK_091654 for RAP2.4 and RAP2.4L, respectively, were obtained from the Arabidopsis stock center. In the case of SALK_093377, homozygous knockout sublines were recovered from the plants whose progeny segregated with 3:1 ratio of kanamycin resistance and kanamycin susceptible seeds. In the case of SALK_091654, the plants were susceptible to kanamycin, and homozygous knockout sublines were recovered after genomic PCR of individual plants. Insertion of the T-DNA into the annotated position was confirmed by genomic PCR and sequencing of the amplified fragments.

\section{Additional material}

Additional file 1: Salt tolerance of AtERF13 oX lines. Plants were germinated and grown on MS medium containing $75 \mathrm{mM}$ or $125 \mathrm{mM}$ $\mathrm{NaCl}$ for 10 days before photographs were taken.

Additional file 2: Mannitol response of RAP2.4L and RAP2.4 OX lines. Plants were germinated and grown on MS medium containing $4 \%$ mannitol for 13 days before photographs were taken. R, RAP2.4 OX lines. RL, RAP2.4L OX lines. Ler, Landsberg erecta.
Additional file 3: Growth of AtERF13, RAP2.4 and RAP2.4L knockout and RNAi lines. RNAi lines of AtERF13 and knockout lines of RAP2.4 and RAP2.4L were prepared as described in the Methods, and their growth phenotypes were investigated. (A) RNAi lines of AtERF13. Top, AtERF13 expression levels determined by RT-PCR. RNA was isolated from plants grown under normal condition. Bottom, plants grown in soil for 25 days. \#25 and \#31 denote RNAi lines. (B) Single or double knockout (KO) lines of RAP2.4 and RAP2.4L. Top left, expression levels of RAP2.4 and RAP2.4L in the single knockout lines of RAP2.4 (RK) and RAP2.4L (RLK) determined by RT-PCR. Top right, expression levels of RAP2.4 and RAP2.4L in the double knockout line (DK). Bottom, plants grown in soil for four weeks.

\section{Acknowledgements}

This work was supported in part by the Korea Research Foundation grant funded by the Korean government (MOEHRD) (KRF-313-2007-2-C00700) and the Mid-career Researcher Program through NRF grant funded by the MEST (No. 2008-0059137). The authors are grateful to the Kumho Life Science Laboratory of Chonnam National University for providing equipments and plant growth facilities.

\section{Authors' contributions}

$\mathrm{SL}$ conducted the expression analysis and analyzed the OX and $\mathrm{KO}$ lines. JHP conducted yeast one-hybrid screens. MHL and JY prepared OX lines and analyzed their phenotypes. SYK designed experiments and wrote the paper. All authors read and approved the final manuscript.

Received: 17 August 2010 Accepted: 16 December 2010 Published: 16 December 2010

\section{References}

1. Finkelstein RR, Gampala SS, Rock CD: Abscisic acid signaling in seeds and seedlings. Plant Cell 2002, 14(Suppl):S15-45.

2. Xiong L, Schumaker KS, Zhu J-K: Cell signaling during cold, drought, and salt stress. Plant Cell 2002, 14(Suppl):S165-183.

3. Hoth S, Morgante M, Sanchez JP, Hanafey MK, Tingey SV, Chua N-H: Genome-wide gene expression profiling in Arabidopsis thaliana reveals new targets of abscisic acid and largely impaired gene regulation in the abi1-1 mutant. J Cell Sci 2002, 115:4891-4900.

4. Takahashi S, Seki M, Ishida J, Satou M, Sakurai T, Narusaka M, Kamiya A, Nakajima M, Enju A, Akiyama K, Yamaguchi-Shinozaki K, Shinozaki K: Monitoring the expression profiles of genes induced by hyperosmotic, high salinity, and oxidative stress and abscisic acid treatment in Arabidopsis cell culture using a full-length cDNA microarray. Plant Mol Biol 2004, 56(1):29-55.

5. Busk PK, Pages M: Regulation of abscisic acid-induced transcription. Plant Mol Biol 1998, 37:425-435.

6. Menkens $A E$, Schindler U, Cashmore AR: The G-box: a ubiquitous regulatory DNA element in plants bound by the GBF family of bZIP proteins. Trend Biochem Sci 1995, 20:506-512.

7. Shen $Q$, Zhang $P, H_{0}$ TH: Modular nature of abscisic acid (ABA) response complexes: composite promoter units that are necessary and sufficient for ABA induction of gene expression in barley. Plant Cell 1996, 8(7):1107-1119.

8. Shen $\mathrm{Q}, \mathrm{Ho} \mathrm{TH}$ : Functional dissection of an abscisic acid (ABA)-inducible gene reveals two independent $A B A$-responsive complexes each containing a G-box and a novel cis-acting element. Plant Cell 1995, 7(3):295-307.

9. Narusaka Y, Nakashima K, Shinwari ZK, Sakuma Y, Furihata T, Abe H, Narusaka M, Shinozaki K, Yamaguchi-Shinozaki K: Interaction between two cis-acting elements, ABRE and DRE, in ABA-dependent expression of Arabidopsis rd29A gene in response to dehydration and high-salinity stresses. Plant J 2003, 34(2):137-148.

10. Choi H, Hong J, Ha J, Kang J, Kim SY: ABFs, a family of ABA-responsive element binding factors. J Biol Chem 2000, 275(3):1723-1730.

11. Uno Y, Furihata $T$, Abe H, Yoshida R, Shinozaki K, Yamaguchi-Shinozaki K: Arabidopsis basic leucine zipper transcription factors involved in an abscisic acid-dependent signal transduction pathway under drought 
and high-salinity conditions. Proc Natl Acad Sci USA 2000, 97(21):11632-11637.

12. Kim SY: The role of ABF family bZIP class transcription factors in stress response. Physiol Plantarum 2006, 126(4):519-527.

13. Finkelstein RR, Lynch TJ: The Arabidopsis abscisic acid response gene ABI5 encodes a basic leucine zipper transcription factor. Plant Cell 2000 12:599-609.

14. Kim SY, Ma J, Perret P, Li Z, Thomas TL: Arabidopsis ABI5 subfamily members have distinct DNA-binding and transcriptional activities. Plant Physiol 2002, 130:688-697.

15. Hobo T, Asada M, Kowyama Y, Hattori T: ACGT-containing abscisic acid response element (ABRE) and coupling element 3 (CE3) are functionally equivalent. Plant J 1999, 19(6):679-689.

16. Niu X, Helentjaris T, Bate NJ: Maize ABI4 binds coupling element1 in abscisic acid and sugar response genes. Plant Cell 2002, 14(10):2565-2575.

17. Shen QJ, Casaretto JA, Zhang P, Ho TH: Functional definition of ABAresponse complexes: the promoter units necessary and sufficient for $A B A$ induction of gene expression in barley (Hordeum vulgare L.). Plant Mol Biol 2004, 54(1):111-124.

18. Nakano T, Suzuki K, Fujimura T, Shinshi H: Genome-wide analysis of the ERF gene family in Arabidopsis and rice. Plant Physiol 2006, 140(2):411-432.

19. Sakuma Y, Liu Q, Dubouzet JG, Abe H, Shinozaki K, Yamaguchi-Shinozaki K: DNA-binding specificity of the ERF/AP2 domain of Arabidopsis DREBs, transcription factors involved in dehydration- and cold-inducible gene expression. Biochem Biophys Res Commun 2002, 290(3):998-1009.

20. Lee SJ, Cho DI, Kang JY, Kim SY: An ARIA-interacting AP2 domain protein is a novel component of ABA signaling. Mol Cells 2009, 27(4):409-416.

21. Feng JX, Liu D, Pan Y, Gong W, Ma LG, Luo JC, Deng XW, Zhu YX: An annotation update via cDNA sequence analysis and comprehensive profiling of developmental, hormonal or environmental responsiveness of the Arabidopsis AP2/EREBP transcription factor gene family. Plant Mol Biol 2005, 59(6):853-868.

22. Jefferson RA, Kavanagh TA, Bevan MW: GUS fusions: $\beta$-glucuronidase as a sensitive and versatile gene fusion marker in higher plants. EMBO J 1987 20:3901-3907.

23. Arenas-Huertero F, Arroyo A, Zhou L, Sheen J, Leon P: Analysis of Arabidopsis glucose insensitive mutants, gin5 and gin6, reveals a central role of the plant hormone $A B A$ in the regulation of plant vegetative development by sugar. Genes Dev 2000, 14(16):2085-2096.

24. Hao D, Ohme-Takagi M, Sarai A: Unique mode of GCC box recognition by the DNA-binding domain of ethylene-responsive element-binding factor (ERF domain) in plant. J Biol Chem 1998, 273(41):26857-26861.

25. Fujimoto SY, Ohta M, Usui A, Shinshi H, Ohme-Takagi M: Arabidopsis ethylene-responsive element binding factors act as transcriptional activators or repressors of GCC box-mediated gene expression. Plant Cell 2000, 12(3):393-404.

26. Lin RC, Park HJ, Wang HY: Role of Arabidopsis RAP2.4 in regulating lightand ethylene-mediated developmental processes and drought stress tolerance. Mol Plant 2008, 1(1):42-57.

27. Dong CJ, Liu JY: The Arabidopsis EAR-motif-containing protein RAP2.1 functions as an active transcriptional repressor to keep stress responses under tight control. BMC Plant Biol 2010, 10:47.

28. Berrocal-Lobo M, Molina A, Solano R: Constitutive expression of ETHYLENE-RESPONSE-FACTOR1 in Arabidopsis confers resistance to several necrotrophic fungi. Plant J 2002, 29(1):23-32.

29. Lorenzo O, Piqueras R, Sanchez-Serrano JJ, Solano R: ETHYLENE RESPONSE FACTOR1 integrates signals from ethylene and jasmonate pathways in plant defense. Plant Cell 2003, 15(1):165-178.

30. McGrath KC, Dombrecht B, Manners JM, Schenk PM, Edgar Cl, Maclean DJ, Scheible WR, Udvardi MK, Kazan K: Repressor- and activator-type ethylene response factors functioning in jasmonate signaling and disease resistance identified via a genome-wide screen of Arabidopsis transcription factor gene expression. Plant Physiol 2005, 139(2):949-959.

31. Ogawa T, Pan L, Kawai-Yamada M, Yu LH, Yamamura S, Koyama T, Kitajima S, Ohme-Takagi M, Sato F, Uchimiya H: Functional analysis of Arabidopsis ethylene-responsive element binding protein conferring resistance to Bax and abiotic stress-induced plant cell death. Plant Physiology 2005, 138(3):1436-1445
32. Onate-Sanchez L, Anderson JP, Young J, Singh KB: AtERF14, a member of the ERF family of transcription factors, plays a nonredundant role in plant defense. Plant Physiol 2007, 143(1):400-409.

33. Pre M, Atallah M, Champion A, De Vos M, Pieterse CM, Memelink J: The AP2/ERF domain transcription factor ORA59 integrates jasmonic acid and ethylene signals in plant defense. Plant Physiol 2008, 147(3):1347-1357.

34. Yang Z, Tian L, Latoszek-Green M, Brown D, Wu K: Arabidopsis ERF4 is a transcriptional repressor capable of modulating ethylene and abscisic acid responses. Plant Mol Biol 2005, 58(4):585-596.

35. Finkelstein RR, Wang ML, Lynch TJ, Rao S, Goodman HM: The Arabidopsis abscisic acid response locus ABI4 encodes an APETALA 2 domain protein. Plant Cell 1998, 10:1043-1054.

36. Kizis D, Pages M: Maize DRE-binding proteins DBF1 and DBF2 are involved in rab17 regulation through the drought-responsive element in an ABA-dependent pathway. Plant J 2002, 30(6):679-689.

37. Lee S-j, Kang J-y, Park H-J, Kim MD, Bae MS, Choi H-i, Kim SY: DREB2C Interacts with ABF2, a bZIP Protein Regulating Abscisic Acid-Responsive Gene Expression, and Its Overexpression Affects Abscisic Acid Sensitivity. Plant Physiol 2010, 153(2):716-727.

38. Song CP, Agarwal M, Ohta M, Guo Y, Halfter U, Wang P, Zhu JK: Role of an Arabidopsis AP2/EREBP-type transcriptional repressor in abscisic acid and drought stress responses. Plant Cell 2005, 17(8):2384-2396.

39. Pandey GK, Grant JJ, Cheong YH, Kim BG, Li L, Luan S: ABR1, an APETALA2-domain transcription factor that functions as a repressor of ABA response in Arabidopsis. Plant Physiol 2005, 139(3):1185-1193.

40. Bechtold N, Pelletier G: In planta Agrobacterium-mediated transformation of adult Arabidopsis thaliana plants by vacuum infiltration. Methods $\mathrm{Mol}$ Biol 1998, 82:259-266.

41. Kang J, Choi H, Im M, Kim SY: Arabidopsis basic leucine zipper proteins that mediate stress-responsive abscisic acid signaling. Plant Cell 2002 14:343-357.

42. Voinnet $O$, Rivas $S$, Mestre $P$, Baulcombe $D$ : An enhanced transient expression system in plants based on suppression of gene silencing by the p19 protein of tomato bushy stunt virus. Plant J 2003, 33(5):949-956.

43. Witte CP, Noel LD, Gielbert J, Parker JE, Romeis T: Rapid one-step protein purification from plant material using the eight-amino acid Strepll epitope. Plant Molecular Biology 2004, 55(1):135-147.

doi:10.1186/1471-2229-10-277

Cite this article as: Lee et al.: Isolation and functional characterization of CE1 binding proteins. BMC Plant Biology 2010 10:277.

\section{Submit your next manuscript to BioMed Central and take full advantage of:}

- Convenient online submission

- Thorough peer review

- No space constraints or color figure charges

- Immediate publication on acceptance

- Inclusion in PubMed, CAS, Scopus and Google Scholar

- Research which is freely available for redistribution

Submit your manuscript at www.biomedcentral.com/submit
C Biomed Central 